\title{
Stable and efficient differential estimators on oriented point clouds
}

\author{
T. Lejemble ${ }^{1}$ (D) and D. Coeurjolly ${ }^{2}$ (D) and L. Barthe ${ }^{1}$ (D) and N. Mellado ${ }^{1}$ (D) \\ ${ }^{1}$ CNRS, IRIT - Université de Toulouse \\ ${ }^{2}$ Université de Lyon, CNRS, LIRIS
}

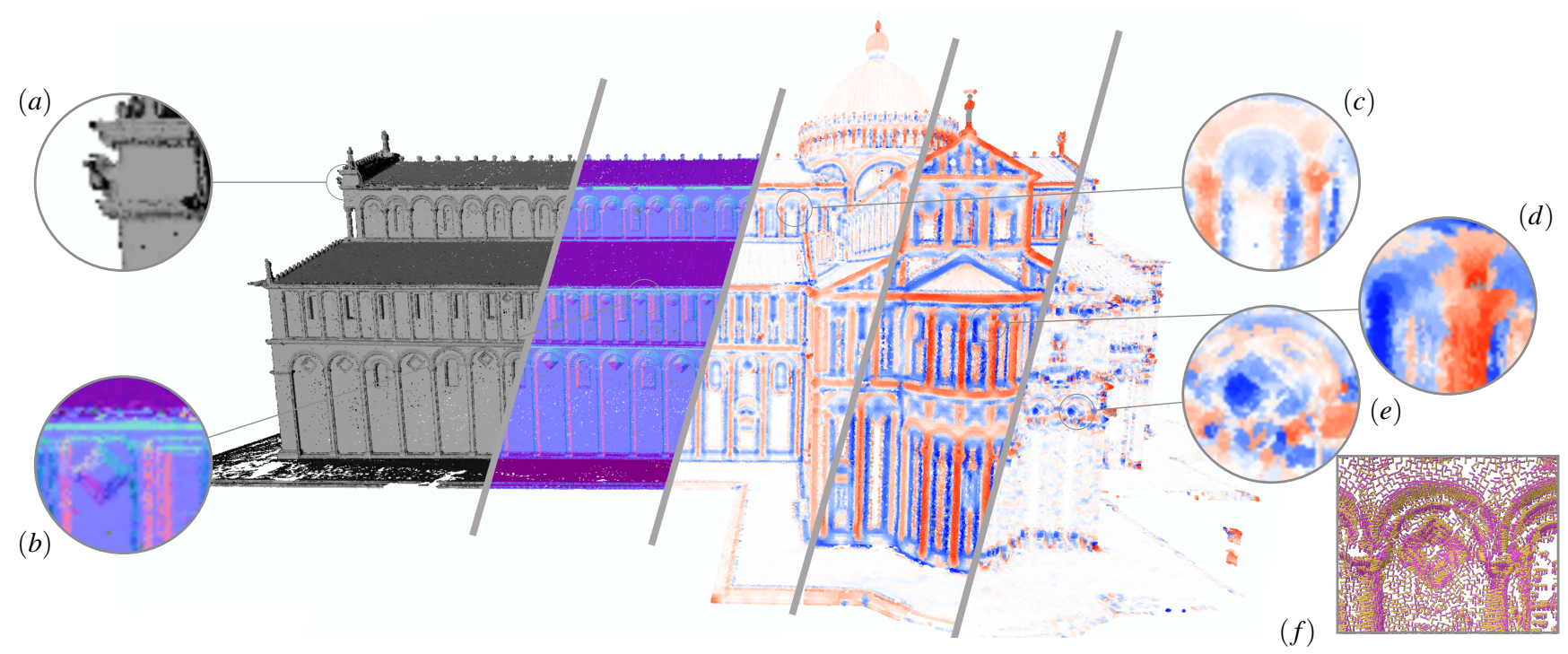

Figure 1: Differential estimations computed with our stable estimators on a large point cloud with normals (2.5M points). Zoom on: (a) the initial point cloud, $(b)$ our corrected normal vectors, $(c)$ mean curvature, $(d, e)$ principal curvatures, and $(f)$ principal curvature directions.

\begin{abstract}
Point clouds are now ubiquitous in computer graphics and computer vision. Differential properties of the point-sampled surface, such as principal curvatures, are important to estimate in order to locally characterize the scanned shape. To approximate the surface from unstructured points equipped with normal vectors, we rely on the Algebraic Point Set Surfaces (APSS) [GG07] for which we provide convergence and stability proofs for the mean curvature estimator. Using an integral invariant viewpoint, this first contribution links the algebraic sphere regression involved in the APSS algorithm to several surface derivatives of different orders. As a second contribution, we propose an analytic method to compute the shape operator and its principal curvatures from the fitted algebraic sphere. We compare our method to the state-of-the-art with several convergence and robustness tests performed on a synthetic sampled surface. Experiments show that our curvature estimations are more accurate and stable while being faster to compute compared to previous methods. Our differential estimators are easy to implement with little memory footprint and only require a unique range neighbors query per estimation. Its highly parallelizable nature makes it appropriate for processing large acquired data, as we show in several real-world experiments.
\end{abstract}

CCS Concepts

- Computing methodologies $\rightarrow$ Computer graphics; Point-based models; Shape analysis; 


\section{Introduction}

Recent developments of technologies and sensors as photogrammetry and Lidar have democratized 3D scanning. 3D point clouds, resulting from these scans, are thus becoming a standard way for acquiring our environment. Their processing is then required for their effective use in many domains of application such as earth and city digitization, culture heritage, engineering, entertainment, health, agriculture, etc. The acquisition pipeline usually starts with the generation of a $3 \mathrm{D}$ point cloud that is challenging to process. These point clouds, composed of a large number of samples (up to billions), exhibit many artefacts, and lack of structure to parameterize the points-sampled surface. This is essentially why traditional signal processing techniques are difficult to apply on unorganized point clouds.

Point cloud processing - as protein surface comparison [Con86], line-based rendering [PKG03], fractured objects reassembly $\left[\mathrm{HFG}^{*} 06\right]$, point cloud registration [GMGP05, MDS15], semantic classification [KHS10, KLM*13, TGDM18] pattern recognition $\left[\mathrm{HLP}^{*} 20\right]$, and real-time point-cloud reconstruction from single photon lidar data [TAM $\left.{ }^{*} 19\right]$ to name a few - often rely on local shape characterization, which may be efficiently done using differential estimations locally describing the unknown surface from the point set. When processing point clouds sampling real-world objects, we thus need accurate (low error), consistent (low variance), robust (stable with respect to noise), and efficient (in time and memory) differential estimators.

Contribution. By relying on the Algebraic Point Set Surfaces (APSS) [GG07] - that efficiently approximate a smooth manifold from scattered points equipped with normal vectors (see Section 3 for a brief background) - we propose to combine two different frameworks to obtain a provable mean curvature estimator and a robust principal curvature estimator on unstructured $3 \mathrm{D}$ point clouds. The analysis of the first, the algebraic sphere regression involved in the APSS algorithm, with the second, an integral invariant viewpoint [MHYS04, PWHY09, DM14], demonstrates convergence and stability guarantees on the mean curvature estimator. In addition, the differentiation of the weighting kernel used in the algebraic sphere fitting, allows us to provide a new analytical formula for the shape operator of the APSS used to derive principal curvatures.

These lead us to the following contributions:

- We perform an asymptotic analysis of the algebraic sphere regression demonstrating that the inverse radius of the fitted sphere tends toward the expected value of the surface mean curvature (Section 4). We also show how the algebraic sphere regression gives access to an anisotropy measure as well as $3^{\text {rd }}$ and $4^{\text {th }}$ order surface derivatives. We analyse the stability by directly linking the strength of a Gaussian noise on positions to the analysis radius of the APSS weighting kernel.

- We analytically differentiate the APSS fitted algebraic sphere to estimate our Algebraic Shape Operator and the principal curvatures of the point-sampled surface (Section 5). We then demonstrate the higher robustness of our Algebraic Shape Operator with several experiments on both synthetic and acquired data in Section 6 .

\section{Related Work}

In this section, we first review the general methods for locally estimating curvatures on unstructured point clouds. We then more specifically present the different methods related to our two frameworks: Point Set Surfaces and Integral Invariants.

Curvatures estimation on point clouds. Pauly et al. [PGK02] introduce the Surface Variation computed from the eigenvalues of the Principal Component Analysis (PCA). It efficiently measures the distance from the surface to a representative plane, but it lacks of efficiency for discriminating between different curved surface. The Osculating Jets method proposed by Cazals and Pouget [CP05] estimates the coefficients of a truncated bi-variate Taylor expansion that best match the neighboring points over the tangent plane. Recently, Béarzi et al. [BDC18] have proposed a similar method decomposing the bivariate Taylor approximation into a radial polynomial and angular oscillations, creating a new set of basis function called Wavejets. These two methods can compute surface derivatives up to any orders at the cost of large systems to solve.

Kalogerakis et al. [KSNS07] directly fit the shape operator of the surface from pairs of neighboring points and their normal vectors. Mérigot et al. [MOG10] estimate principal curvatures from Voronoi Covariance Measures (VCM) computed from the Voronoi diagram of the point cloud. Although this method comes with theoretical guarantees, it remains relatively sensitive in practice when there is a significant amount of noise and varying sampling as usually found in real-world acquired data. Guerrero et al. [GKOM18] propose a deep neural network called PCPNet, which is inspired from the pioneer PointNet architecture [QSMG17]. Such machine learning approach experimentally gives accurate estimations of principal curvatures, but guarantees on the accepted level of noise and sampling variation are missing.

Point Set Surfaces. Introduced by Alexa et al. [ABCO*01], Point Set Surfaces (PSS) approaches use Moving Least Squares regressions [Lev98, Lev04] for approximating a smooth manifold from scattered points. This local procedure estimates a reference plane using non-linear optimization and fits a bivariate polynomial to the local neighborhood expressed over it. A simpler and more efficient PSS is provided with an implicit formulation [AA03] using weighted average position and PCA. Another similar but slightly more general PSS proposed by Amenta et al. [AK04] is defined by the critical points of an energy function along lines determined by a given vector field. Principal curvatures are directly available from such PSS formulation [YQ07]. Many other versions exist including Progressive PSS [FCOAS03] that build a multi-resolution surface, Anisotropic PSS [AA06], which are more robust to irregular sampling, and Parabolic-cylindrical PSS [RGRG15] enforcing developability. Guennebaud and Gross [GG07] propose to directly fit an algebraic sphere [Pra87a] to avoid the critical step that estimates a reference plane. These Algebraic Point Set Surfaces (APSS) are stable in presence of noise and are efficient to compute thanks to the closed-form formula of the algebraic sphere regression [GGG08]. The inverse radius of the fitted sphere provides an estimate for the mean curvature. The APSS is a classical method developed in the context of point cloud rendering, but it is also used to analyse the point-sampled surface as done by Mellado et al. [MGB*12] with 
the Growing Least Squares (GLS). They provide point-wise multiscale differential descriptors based on the APSS that are widely used for point cloud registration [MDS15], modeling [NGM14], and pattern recognition [LMBM20, HLP $\left.{ }^{*} 20\right]$. In addition to its efficiency and robustness, we rely on the APSS for the analytical formula of the sphere fitting, which makes it suitable for an integral invariant analysis and for kernel differentiation.

Integral invariants. Integral invariants refers to a family of approaches that provably links integral operations on local geometric quantities around a point on a surface to its differential properties. This fundamental link is theoretically proven using an asymptotic setting where the size of the neighborhood tends toward zero. Manay et al. [MHYS04] introduced this name to design local signatures for $2 \mathrm{D}$ shape matching. Their work follow the longstanding research on the connections that exist between locally integrated measures like area, volumes and moments with surface curvatures [Ber48, Con86, HT03, CRT04]. More recently, Pottman et al. [PWY*07, PWHY09] provide several methods to compute various integral invariants on meshes. They obtain principal curvatures estimations [YLHP06] that are shown to be more robust than those obtained using PCA [CRT04,PKG03], normal cycles [CSM03] and Osculating Jets [CP05]. Digne et al. [DMSL11] leverage the PCA to perform a mean curvature flow for point cloud smoothing. Integral invariants can be formulated as soon as local sums are computed around a point as done by Digne et al. [DM14] for several curvature estimators [BC94, LT90, PGK02] and regression methods [DMSL11, ABCO*01]. The integral nature of such approaches makes them particularly stable with respect to noise. For this reason, they are used for many geometry processing tasks such as point cloud registration [GMGP05], shape matching $\left[\mathrm{HFG}^{*} 06\right]$, shape editing [ $\left.\mathrm{LZH}^{*} 07\right]$, as well as digital geometry analysis [CLL13].

\section{Background: Algebraic Point Set Surfaces}

We first remind the algebraic sphere regression involved in the APSS [GG07]. Instead of directly fitting a more general quadric [AJB* 07], the algebraic sphere regression does not lead to any ambiguous geometric configuration (e.g. near double sheets surfaces), usually involves less computational resources, and still remains accurate for principal curvatures estimation as we will show. The algebraic sphere is represented as the 0-isosurface of the following scalar field function:

$$
f(\mathbf{x}):=u_{c}+\mathbf{u}_{\ell}^{T} \mathbf{x}+u_{q} \mathbf{x}^{T} \mathbf{x},
$$

where $u_{c} \in \mathbb{R}, \mathbf{u}_{\ell} \in \mathbb{R}^{3}$ and $u_{q} \in \mathbb{R}$ are respectively the constant, linear and quadratic coefficients of the sphere. Fitting an algebraic sphere to $N$ oriented points $\left\{\mathbf{p}_{i}, \mathbf{n}_{i}\right\}_{i=1 \ldots N}$ leads to the closed-form solution [GGG08, Equation 6]:

$$
\begin{aligned}
& u_{q}(\mathbf{x})=\frac{1}{2} \frac{\sum_{i} w_{i} \mathbf{p}_{i} \cdot \mathbf{n}_{i}-\sum_{i} w_{i} \mathbf{p}_{i} \cdot \sum_{i} w_{i} \mathbf{n}_{i}}{\sum_{i} w_{i} \mathbf{p}_{i} \cdot \mathbf{p}_{i}-\sum_{i} w_{i} \mathbf{p}_{i} \cdot \sum_{i} w_{i} \mathbf{p}_{i}} \\
& \mathbf{u}_{\ell}(\mathbf{x})=\frac{1}{\sum_{i} w_{i}}\left(\sum_{i} w_{i} \mathbf{n}_{i}-2 u_{q}(\mathbf{x}) \sum_{i} w_{i} \mathbf{p}_{i}\right), \\
& u_{c}(\mathbf{x})=-\frac{1}{\sum_{i} w_{i}}\left(\mathbf{u}_{\ell}(\mathbf{x}) \cdot \sum_{i} w_{i} \mathbf{p}_{i}+u_{q}(\mathbf{x}) \sum_{i} w_{i} \mathbf{p}_{i} \cdot \mathbf{p}_{i}\right),
\end{aligned}
$$

where $w_{i}=w_{r}\left(\mathbf{p}_{i}-\mathbf{x}\right)$ is a weighting function of support size $r \in$ $\mathbb{R}^{+}$defined by:

$$
w_{r}(\mathbf{x}):=K\left(\frac{\|\mathbf{x}\|}{r}\right) .
$$

The smooth decreasing kernel is typically defined by the polynomial $K(x)=\left(x^{2}-1\right)^{2}$.

As pointed by Mellado et al. [MGB*12], a given set of parameters $u_{c}, \mathbf{u}_{\ell}$ and $u_{q}$ characterizes an infinite set of local hyperspheres as there exists an infinity of scalar fields (based on scalar multiples of $\left.\left[u_{c}, \mathbf{u}_{\ell}, u_{q}\right]\right)$ that correspond to the same fitting coefficients. To solve this issue and consistently pick a unique solution, they use Pratt's normalization [Pra87b] to constrain the scalar field to have a unitary gradient vector on the 0 -isosurface, yielding:

$$
\hat{u}_{c}=\frac{u_{c}}{p(\mathbf{x})}, \quad \hat{\mathbf{u}}_{\ell}=\frac{\mathbf{u}_{\ell}}{p(\mathbf{x})}, \quad \hat{u}_{q}=\frac{u_{q}}{p(\mathbf{x})},
$$

with $p:=\sqrt{\left\|\mathbf{u}_{\ell}\right\|^{2}-4 u_{c} u_{q}}$ being the Pratt's norm. Two differential estimators can be derived from these normalized parameters [GG07] computed without loss of generality at the origin of $\mathbb{R}^{3}$ : the mean curvature $\tilde{H}$ and the corrected normal vector $\tilde{\mathbf{n}}$, that are respectively defined as:

$$
\begin{aligned}
\tilde{H} & :=2 \hat{u}_{q}, \\
\tilde{\mathbf{n}} & :=\frac{\hat{\mathbf{u}}_{\ell}}{\left\|\hat{\mathbf{u}}_{\ell}\right\|} .
\end{aligned}
$$

$\tilde{H}$ corresponds to the inverse radius of the hypersphere, and $\tilde{\mathbf{n}}$ to the normalized gradient. We present in Section 4 a study of the asymptotic behavior of differential quantities estimated from the algebraic hypersphere parameters, and we introduce in Section 5 a novel estimator for the shape operator.

\section{Differential properties from the algebraic sphere regression}

This section is organized as follows. We first describe the asymptotic analysis framework in Section 4.1 that is used to recover stable differential quantities from the hypersphere coefficients (Sections 4.2 and 4.3). Then, we study the asymptotic properties of other estimators derived from the hypersphere coefficients: the spatial derivatives of the coefficients (Section 4.4) and the bilaplacian flow (Section 4.5). We introduce in Section 4.6 a theoretical analysis of the estimators robustness when the input point samples are perturbed by Gaussian noise. Proofs are detailed in the supplementary material.

\subsection{Asymptotic framework}

We study a smooth regular surface $S$ embedded in $\mathbb{R}^{3}$, and the analysis focuses on one of its point $\mathbf{p} \in S$ and its neighborhood within a fixed distance $r \in \mathbb{R}^{+}$. The frame of analysis is the so-called principal frame [PWY* 07] (also called local canonical frame [DC76, Section 1.6] or local intrinsic coordinate system [DMSL11]). The frame origin is defined such that $\mathbf{p}=\mathbf{0}$, and the surface $S$ is locally expressed as a height field over its tangent plane by using the mapping:

$$
\mathbf{f}(x, y):=\left[\begin{array}{lll}
x & y & z(x, y)
\end{array}\right]^{T} .
$$


T. Lejemble \& D. Coeurjolly \& L. Barthe \& N. Mellado / Stable and efficient differential estimators on oriented point clouds

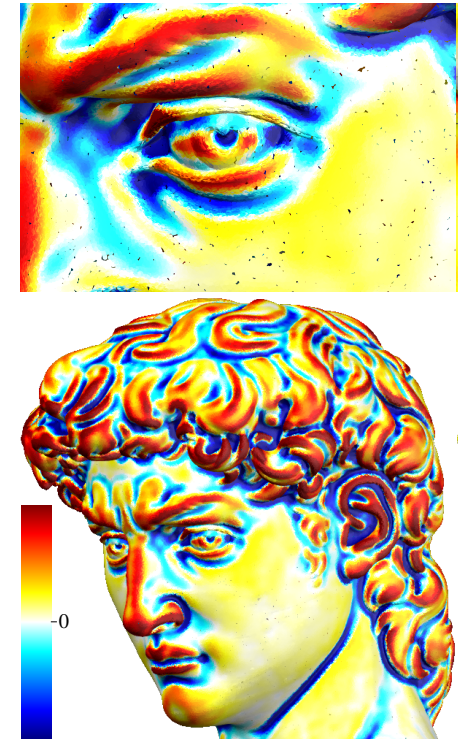

(a) $\hat{u}_{q} \sim H$

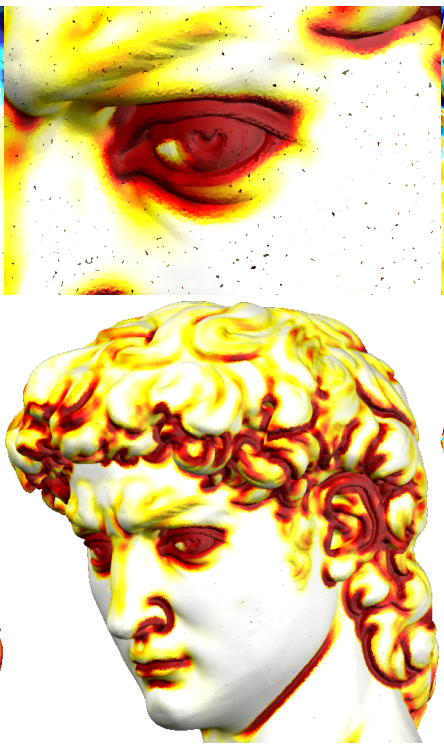

(b) $1-p \sim\left(\kappa_{1}-\kappa_{2}\right)^{2}$

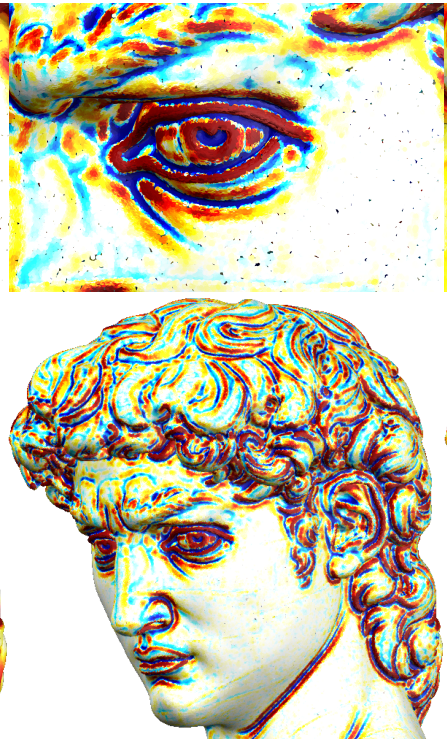

(c) $\hat{u}_{c} \sim 9 H^{3}-5 K H-\Delta H$

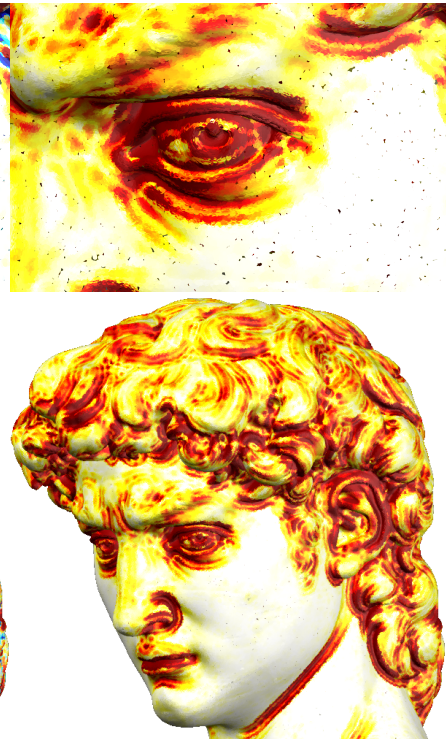

(d) $\sqrt{v} \sim\|\nabla H\|$

Figure 2: Geometric features: obtained from local algebraic sphere regressions performed on 2 million unstructured points (dark dots are due to the splats rendering). We summarize the relevant differential properties that are asymptotically linked to each of them by the symbol $\sim$. (a) The quadratic parameter is related to the mean curvature $H$ (Theorem 1). (b) The deviation of the Pratt's norm from 1 defines an anisotropy measure (Proposition 1). (c) The constant parameter involves $4^{\text {th }}$ order derivatives which highlights fine surface variations (Theorem 1). (d) The squared root of the geometric variation [MGB ${ }^{*}$ 12] is related to the norm of the mean curvature gradient involving $3^{\text {rd }}$ order derivatives (Proposition 2).

The coordinates $x$ and $y$ on the plane are aligned with the directions of principal curvatures $\kappa_{1}$ and $\kappa_{2}$. The height $z$ along the surface normal vector $\mathbf{n}$ is given by the following Taylor expansion of order 5 (minimum order required for the following studies):

$$
z(x, y)=\sum_{k=0}^{5} \sum_{j=0}^{k}\left(\begin{array}{l}
k \\
j
\end{array}\right) \frac{x^{j} y^{k-j}}{k !} a_{j, k-j}+O\left(x^{6}+y^{6}\right) .
$$

The coefficients $a_{j, k-j}=\frac{\partial^{k} z}{\partial x^{j} \partial y^{k-j}}$ correspond to the successive derivatives of $z$ evaluated at $\mathbf{p}$. In this work, we focus on the following differential quantities: the principal curvatures that are explicitly written as $\kappa_{1}=a_{20}$ and $\kappa_{2}=a_{02}$, and the mean and Gaussian curvature that are respectively denoted by $H=\left(\kappa_{1}+\kappa_{2}\right) / 2$ and $K=\kappa_{1} \kappa_{2}$. Note that this local principal frame is chosen so that $a_{00}=a_{10}=a_{01}=a_{11}=0$. The Laplace operator applied to the mean curvature, which is also half of the bilaplacian of $z$, is also explicitly denoted by $\Delta H=\frac{1}{2} \Delta^{2} z=\frac{1}{2}\left(a_{40}+2 a_{22}+a_{04}\right)$.

In this smooth setting, any discrete sum appearing in Equations (2)-(4) is replaced by an integral over the surface patch: $\mathcal{P}_{r}:=\mathcal{B}_{r}(\mathbf{p}) \cap S$, where $\mathcal{B}_{r}(\mathbf{p})$ is the ball of center $\mathbf{p}$ and radius $r$. Integrating over $\mathcal{P}_{r}$ is actually asymptotically equivalent to integrating over the cylindrical neighborhood [DMSL11, Lemma 1]:

$$
\mathcal{D}_{r}:=\left\{(x, y) \in \mathbb{R}^{2}, x^{2}+y^{2}<r^{2}\right\},
$$

which is used instead as it makes analytical integration possible. Note that for simplicity, this study considers a constant weighting $w_{i}=1$ instead of the smooth decreasing weighting kernel of Equation (5) that is used in practice. Apart from changing multiplicative constants, this modification does not impact the results of the following sections.

\subsection{Algebraic sphere coefficients}

Our first result stated in Theorem 1 concerns the link between the coefficients of the normalized algebraic sphere (see Equation (6)) and the differential properties of the surface.

Theorem 1 The parameters of the algebraic sphere fitted to the surface patch $\mathcal{P}_{r}$ have the following asymptotic expansions:

$$
\begin{aligned}
\hat{u}_{q} & =-\frac{H}{2}+O(r), \\
\hat{\mathbf{u}}_{\ell} & =\left[\begin{array}{l}
0 \\
0 \\
1
\end{array}\right]-\left[\begin{array}{c}
\frac{a_{30}+a_{12}}{8} \\
\frac{a_{03}+a_{21}}{8} \\
0
\end{array}\right] r^{2}+O\left(r^{3}\right), \\
\hat{u}_{c} & =-\frac{1}{96}\left(9 H^{3}-5 K H-\Delta H\right) r^{4}+O\left(r^{5}\right) .
\end{aligned}
$$

The full proof is provided in the supplementary material. Equation (12) demonstrates that the quadratic coefficient $\hat{u}_{q}$ is proportional to the mean curvature of the surface as visually validated in Figure 2-(a). In case the surface is flat, the mean curvature $H$ is null, $\hat{u}_{q}$ is equal to zero, and the fitted algebraic sphere of Equation (1) degenerates to a plane. The constant parameter $\hat{u}_{c}$ is the algebraic distance between the point of analysis and the algebraic sphere. It 
is asymptotically equivalent to a mix between the mean and Gaussian curvatures, and the bilaplacian of the surface $\Delta H$. Since this last quantity involves $4^{\text {th }}$ order surface derivatives, $\hat{u}_{c}$ highlights the fine variations of the surface as shown in Figure 2-(c).

\subsection{APSS differential estimators}

We now give one of our key contribution, which is the convergence proof of the APSS differential estimators $\tilde{H}$ and $\tilde{\mathbf{n}}$ (Equations (7) and (8)).

Corollary 1 The normal estimator $\tilde{\mathbf{n}}$ and the mean curvature estimator $\tilde{H}$ have the following asymptotic expansion:

$$
\begin{aligned}
\tilde{\mathbf{n}} & =\left[\begin{array}{l}
0 \\
0 \\
1
\end{array}\right]-\left[\begin{array}{c}
\frac{a_{30}+a_{12}}{8} \\
\frac{a_{03}+a_{21}}{8} \\
0
\end{array}\right] r^{2}+O\left(r^{3}\right), \\
\tilde{H} & =-H+O(r) .
\end{aligned}
$$

This corollary follows exactly from Equations (7) and (8) and Equations (12)-(14) of Theorem 1.

\subsection{Other curvature-related estimators}

Even though the algebraic sphere is, by definition, isotropic and cannot estimate principal curvatures, it can still measure the anisotropy of the principal curvatures, as detailed in the following proposition.

Proposition 1 The Pratt's norm is asymptotically given by

$$
p=1-\frac{\left(\kappa_{1}-\kappa_{2}\right)^{2}}{16} r^{2}+O\left(r^{3}\right) .
$$

In other words, the Pratt's norm deviates from 1 by a quantity proportional to $\left(\kappa_{1}-\kappa_{2}\right)^{2}$. Therefore, the difference $1-p$ can be used to define an anisotropy measure of the surface. As we can see in Figure 2-(b), it is null around flat and spherical regions, whereas it takes high values near elongated features.

Also, the spatial derivatives of the hypersphere coefficients appear in the geometric variation $v$ introduced by Mellado et al. [MGB* 12, Equation 5]. It measures the variation of the algebraic hypersphere when the neighborhood size $r$ changes:

$$
v:=\left(\partial_{r} \hat{u}_{c}\right)^{2}+\left(r \partial_{r} \tilde{\mathbf{n}}\right)^{2}+\left(r^{2} \partial_{r} \tilde{H}\right)^{2}
$$

Proposition 2 The asymptotic expansion of the GLS geometric variation is

$$
v=\frac{\|\nabla H\|^{2}}{4} r^{4}+O\left(r^{5}\right)
$$

where $\nabla H=\left[\begin{array}{ll}\frac{a_{30}+a_{12}}{2} & \frac{a_{03}+a_{21}}{2}\end{array}\right]^{T}$.

Therefore $v$ is related to the norm of the spatial gradient of the mean curvature $H$ and thus involves $3^{\text {rd }}$ order surface derivatives. Figure 2-(d) shows that the GLS geometric variation characterizes detailed geometric features, which remain different from those characterized from $\hat{u}_{c}$ and $\hat{u}_{q}$ (Theorem 1).

\subsection{Bilaplacian flow estimator}

This section presents the projection operator sending the point of analysis $\mathbf{p}$ onto the algebraic sphere that is fitted to the surface patch $\mathcal{P}_{r}$ around it.

The projection of a point $\mathbf{x}$ onto the sphere is defined by:

$$
\varphi(\mathbf{x})= \begin{cases}\mathbf{x}-\frac{f(\mathbf{x})}{\|\nabla f(\mathbf{x})\|^{2}} \nabla f(\mathbf{x}) & \text { if } u_{q}=0, \\ \mathbf{x}-\frac{\|\nabla f(\mathbf{x})\|^{-p}}{2 u_{q}\|\nabla f(\mathbf{x})\|} \nabla f(\mathbf{x}) & \text { otherwise. }\end{cases}
$$

In the asymptotic settings introduced in Section 4.1, the projection of the center point $\mathbf{p}$ amounts to:

$$
\varphi= \begin{cases}-\frac{\hat{u}_{c}}{\left\|\hat{\mathbf{u}}_{\ell}\right\|} \tilde{\mathbf{n}} & \text { if } u_{q}=0, \\ -\frac{\left\|\hat{\mathbf{u}}_{\ell}\right\|}{2 \hat{u}_{q}} \tilde{\mathbf{n}} & \text { otherwise. }\end{cases}
$$

Proposition 3 The projection of the point of analysis onto the fitted algebraic sphere is

$$
\varphi=\frac{1}{96}\left(9 H^{3}-5 K H-\Delta H\right) r^{4} \mathbf{n}+O\left(r^{5}\right) .
$$

The projection onto the fitted algebraic sphere displaces the point along the normal vector $\mathbf{n}$ with a distance proportional to $9 H^{3}-$ $5 K H-\Delta H$. Since $\Delta H$ appears in this equation, repeating several iterations of such algebraic sphere projections creates a bilaplacian flow. The geometric flow induced by this projection operator is to algebraic sphere regression what the mean curvature flow is to PCA-based plane regression [DMSL11]. In addition, it can be related to the bilaplacian flow implied by the PSS [ABCO*01] as demonstrated by Digne et al. [DM14].

In Figure 3, we compare the PCA-based mean curvature flow with our algebraic sphere-based bilaplacian flow. After tens of iterations, our flow gives a more rounded shape and avoids the critical instabilities of the mean curvature flow that are due to singularities appearing near the medial axis of the surface.

\subsection{Stability analysis}

In this section we study the convergence behaviors of the APSS estimators (Equations (7) and (8)) when the 3D points are perturbed by noise. More formally, each 'true' positions $\mathbf{p}$ is modified by a noise displacement $3 \mathrm{D}$ vector $\boldsymbol{\varepsilon}$ :

$$
\mathbf{p}^{\star}=\mathbf{p}+\boldsymbol{\varepsilon} .
$$

$\boldsymbol{\varepsilon} \sim \mathcal{N}_{3}\left(0, \sigma^{2}\right)$ is an isotropic Gaussian noise with an expected value equal to zero, and with a standard deviation given by:

$$
\sigma=\delta r^{\beta}+O\left(r^{\beta+1}\right)
$$

defined by a constant $\delta>0$, and an order $\beta>0$. In the following we report an appropriate order $\beta$ that is sufficient to ensure that the perturbed estimators $\tilde{H}^{\star}$ and $\tilde{\mathbf{n}}^{\star}$ are convergent.

Theorem 2 For $\beta>2$, we have

$$
\begin{aligned}
\tilde{\mathbf{n}}^{\star}=\left[\begin{array}{l}
0 \\
0 \\
1
\end{array}\right]-\left[\begin{array}{c}
\frac{a_{30}+a_{12}}{8} \\
\frac{a_{03}+a_{21}}{8} \\
0
\end{array}\right] r^{2}+O\left(r^{3}\right), \\
\tilde{H}^{\star}=-H+O(r) .
\end{aligned}
$$



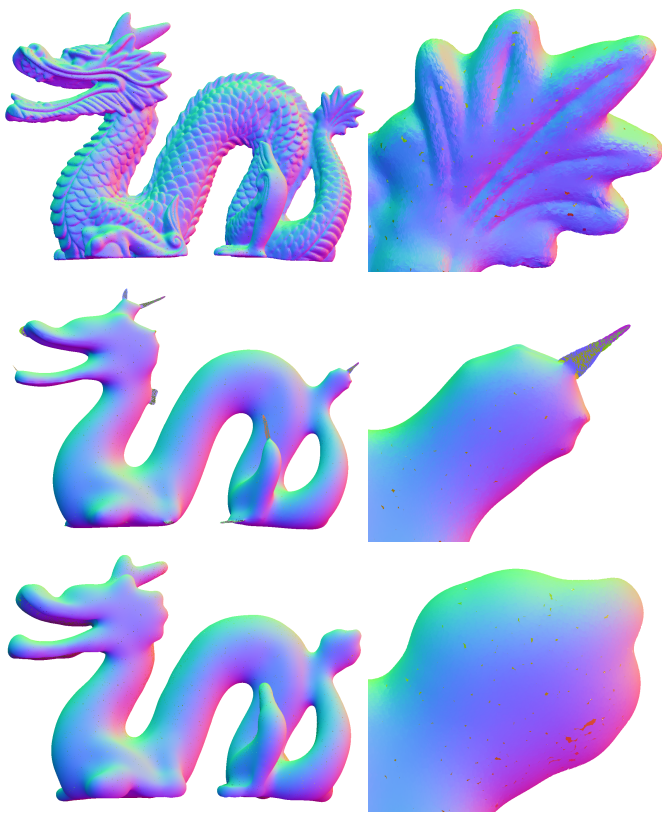

Figure 3: Geometric flows: after 30 iterative projections onto PCA planes (middle) and algebraic spheres (bottom) using the initial point cloud shown on the top.

This stability theorem shows that the normal and the mean curvature estimators converge toward their theoretical values as long as the Gaussian noise standard deviation $\sigma$ has at least a quadratic relation with the regression radius $r$. For $\beta \leq 2$, these estimators are biased by the noise and do not converge toward $\mathbf{n}$ and $H$. All proofs are given in the supplementary material.

\section{Algebraic shape operator}

Our second contribution concerns the estimation of the principal curvatures from the algebraic sphere fitting. The goal is to compute the shape operator:

$$
W:=P \frac{\nabla^{2} f}{\|\nabla f\|} P,
$$

of the scalar field function $f$ of Equation (1), where $P$ is the 3-by2 transfer matrix from the $2 \mathrm{D}$ tangent plane to the $3 \mathrm{D}$ space. We recall that for any unit direction $\mathbf{v}=\left[\begin{array}{ll}u & v\end{array}\right]^{T}$ defined on the tangent plane, applying the shape operator as $\mathbf{v}^{T} W \mathbf{v}$ gives the normal curvature of the surface in that direction.

Using only Equation (1), and thus considering $u_{c}, \mathbf{u}_{\ell}$ and $u_{q}$ as constants, leads to a symmetric shape operator from which principal curvatures cannot be accurately computed. Instead, we propose to rely on the smoothing kernel of Equation (5) used in the weighted Least Squares algebraic sphere regression in order to differentiate the fitted scalar field of the APSS. Indeed, the parameters of the fitted sphere given in Equations (2)-(4) actually depend on $\mathbf{x}$ through the weighting kernel that can be differentiated twice.

From the fitted algebraic sphere, we obtain the following gradi- ent:

$$
\nabla f(\mathbf{x})=\nabla u_{c}+\mathbf{u}_{\ell}+\nabla \mathbf{u}_{\ell}^{T} \mathbf{x}+2 u_{q} \mathbf{x}+\nabla u_{q} \mathbf{x}^{T} \mathbf{x},
$$

and the following Hessian matrix:

$$
\begin{aligned}
\nabla^{2} f(\mathbf{x})= & \nabla^{2} u_{c}+\nabla \mathbf{u}_{\ell}+\nabla \mathbf{u}_{\ell}^{T}+\nabla^{2} \mathbf{u}_{\ell} \mathbf{x}+2 \nabla u_{q} \mathbf{x}^{T} \\
& +\mathbf{x}^{T} \mathbf{x} \nabla^{2} u_{q}+2 u_{q} I_{3}+2 \mathbf{x} \nabla u_{q}^{T},
\end{aligned}
$$

which, when combined with Equation (27), define our Algebraic Shape Operator (ASO). Note that each expression in Equations (28) and (29) depends on $\mathbf{x}$, which is omitted for clarity. Since $\mathbf{u}_{\ell}=\left[\begin{array}{lll}\mathbf{u}_{\ell x} & \mathbf{u}_{\ell y} & \mathbf{u}_{\ell z}\end{array}\right]^{T}$ is a 3D vector, the term $\nabla^{2} \mathbf{u}_{\ell} \mathbf{x}$ in Equation (29) is the product between the rank-3 tensor $\nabla^{2} \mathbf{u}_{\ell}$ and the 3-dimensional vector $\mathbf{x}=\left[\begin{array}{lll}x & y & z\end{array}\right]^{T}$, which gives:

$$
\nabla^{2} \mathbf{u}_{\ell} \mathbf{x}=x \nabla^{2} \mathbf{u}_{\ell x}+y \nabla^{2} \mathbf{u}_{\ell y}+z \nabla^{2} \mathbf{u}_{\ell z} .
$$

By denoting $\lambda_{1}$ and $\lambda_{2}$ the two eigenvalues, and $\mathbf{e}_{1}$ and $\mathbf{e}_{2}$ the two eigenvectors of the shape operator $W$, we obtain the following estimators for the normal vector, the mean, Gaussian, and principal curvatures, and the principal directions at the point from where the APSS algebraic sphere has been fitted:

$$
\begin{aligned}
\tilde{\mathbf{n}}_{w} & :=\frac{\nabla f(\mathbf{x})}{\|\nabla f(\mathbf{x})\|}, \\
\tilde{H}_{w} & :=\frac{\lambda_{1}+\lambda_{2}}{2}, \\
\tilde{K}_{w} & :=\lambda_{1} \lambda_{2}, \\
\tilde{\kappa}_{1} & :=\lambda_{1}, \quad \tilde{\kappa}_{2}:=\lambda_{2}, \\
\mathbf{d}_{1} & :=e_{1}, \quad \mathbf{d}_{2}:=e_{2} .
\end{aligned}
$$

The analytical Equations (28) and (29) can be implemented in the same unique loop over the neighboring points used for the fitting, and without significant additional memory. It results in an efficient algebraic shape operator (ASO) leading to principal curvatures that are evaluated in the next section.

\section{Experimental Evaluation}

In this section, we compare our estimators with previous methods. More precisely, we consider the estimators listed in Table 1 to evaluate either the mean curvature, or the complete curvature tensor on point clouds. All methods have been implemented in the same framework (detailed in Section 6.3) that will be made publicly available.

To evaluate the stability of the estimators with respect to a ground truth, we consider the isosurface of a polynomial implicit function sampled using a Lloyd's relaxation scheme [Lev15]. This allows us to compare the estimated differential quantities with respect to the expected ones, for various sample counts $N$. All methods in Table 1 have a unique parameter: the radius $r$ of the Euclidean ball used to define the local neighborhood of a given point. The VCM approach has a second convolution parameter $R$ that has been set as a fraction of $r$ as suggested in [PC21] (i.e. $R=0.5 r$ ). Compared to other approaches, the APSS and ASO estimators require oriented points, thus with prescribed normal vectors. Depending on the experiments, the normal vector field can be either 
given by the acquisition device (Lidar setting), by an initial rough estimation (for instance using a PCA fitting of the neighborhood), or from the underlying smooth surface (e.g. available in the convergence test). As a consequence, except for APSS and ASO, all mean curvature estimators listed in Table 1 output absolute value of the mean curvature.

\subsection{Convergence experiments}

In this first test, we consider a smooth surface, the Goursat 0surface defined by $-8-2 x^{2}+0.03 x^{4}-2 y^{2}+0.03 y^{4}-2 z^{2}+$ $0.03 z^{4}=0$, which exhibits an interesting range of curvature values, and evaluate the differential estimators on oriented points $\left\{\mathbf{p}_{i}, \mathbf{n}_{i}\right\}$ sampling the isosurface (using the above-mentioned Lloyd's relaxation approach). We first evaluate the convergence of the mean curvature and corrected normal vector quantities while increasing the number of points $N$. In this setting, we have considered a fixed radius $r$ set to $5 \%$ of the limit shape diagonal. Figure 4 presents logscale graph of the estimation error for the absolute value of the estimated mean curvature $\left(\frac{1}{N} \sum_{i}\left(\left|H_{\text {estimated }}\right|-\left|H_{\text {expected }}\right|\right)\right)$, as well as the corrected normal vector estimation $\left(\frac{1}{N} \sum_{i}\left(1-\mid \mathbf{n}_{\text {estimated }}\right.\right.$. $\left.\mathbf{n}_{\text {expected }} \mid\right)$ ), with respect to $N$.

First, we observe a good experimental convergence of WJets, OJets, PSS, and the proposed estimators, which matches with the theoretical guarantees provided by these methods (Corollary 1 for APSS). We also observe a very good experimental behavior of the quantities derived from our curvature tensor ASO, namely $\tilde{H}_{w}$ and $\tilde{\mathbf{n}}_{w}$.

\subsection{Robustness experiments}

We evaluate the robustness of the estimated quantities with respect to noise, either on the position or the prescribed normal vector field. In Figure 5 and to match with the statement of Theorem 2, we compare our approaches with previous ones when a Gaussian noise on the positions $\left\{\mathbf{p}_{i}\right\}$ is considered (with standard deviations in $\{0,0.005,0.01,0.02\}$ ). In all these tests, we set the radius parameter to $r=0.1$. For each result, we render the absolute value of the mean curvature estimator (top-left part) and a pure reflexive material with a zebra pattern to highlight the smoothness of the corrected normal vector (bottom-right part). Although a Gaussian noise is not a complete representative model of a real acquisition noise, it can still be shared by many 3D acquisition sensors and techniques. In the supplementary material, we provide additional robustness tests on non-uniform sampling of the input geometry, as well as a simulated Lidar sampling with noise in the depth measurement. We also provide further experiments with various $r$ (which can also be set as a function of the noise level) and the estimators proposed in [PWY* 07], [DMSL11] and [GKOM18] (for both single scale and multi-scale pre-trained networks).

Figure 5 also highlights a robustness test with respect to the input normal vector field $\left\{\mathbf{n}_{i}\right\}$ : if we consider an estimation of the normal vector field using a simple PCA fitting $\left(\left\{\mathbf{n}_{P C A}\right\}+\right.$ APSS and $\left\{\mathbf{n}_{P C A}\right\}+$ ASO), we observe similar results on this object compared to exact normal vectors from the implicit surface. In Figure 6, we evaluate the stability of the APSS and ASO quantities when the input normal vectors are perturbed using a varying angular random noise of $10^{\circ}, 20^{\circ}$ and $30^{\circ}$. We have also performed an additional test when normal vectors are flipped with probabilities 0.1 and 0.3 . We observe a strong stability of both APSS and ASO quantities, with an even better stability for A.SO.

Finally, Figures 1 and 7 illustrate various differential quantities (mean curvature, corrected normal vectors, Gaussian curvature, principal curvature values and directions) on a Lidar $\mathrm{P}$ is a data set (2 506408 points) and the Dragon statue (127 572 points).

\subsection{Implementation details and timings}

All the methods have been implemented in $\mathrm{C}++$ using Eigen [GJ*10], except for VCM and OJets whose implementations come from CGAL [PC21, AGJ*21]. The APSS code comes from Ponca [MLGB20], and we converted in C++ the Matlab implementation of WJets released by the authors. Point cloud renderings have been obtained using Radium $\left[\mathrm{MRB}^{*}\right]$. The same k-d tree is used to accelerate range neighbors queries (except for $\mathrm{VCM}$ that builds a Voronoi diagram). Its branches split across the most elongated dimension at the middle, and it has a maximal depth of 32 and a maximum of 64 points per leaf. The proposed estimators will be released in the Ponca library [MLGB20] upon publication.

Timings reported in Table 2 are recorded using an Intel(R) Xeon(R) CPU with 40 cores at $2.40 \mathrm{GHz}$ and $128 \mathrm{G}$ of RAM. Our shape operator (ASO) adds a slight overhead compared to APSS due to the computation of Equations (28) and (29). It remains faster than OJets, WJets, PSS and VCM since it avoids the decomposition of large matrices and the construction of a Voronoi diagram. In addition, our method has a constant complexity in memory with respect to the number of neighbors, contrary to OJet s and WJets. Computing the distance to the barycenter [PWY* 07] and to the PCA plane [DMSL11] is very fast, but it is limited to mean curvature estimations and gives imprecise results in practice. To summarize, our method is the fastest and the most accurate and robust way (Sections 6.1 and 6.2) to estimate principal curvatures in unstructured point clouds.

\section{Conclusions}

We have introduced an analysis of the theoretical stability properties of the differential estimators based on the APSS. From a computational point of view, the proposed estimators can easily be implemented on parallel architectures, only requiring nearest neighbor queries for which many efficient data structures exist. We have shown that these quantities converge to the expected differential ones (e.g. mean curvature, corrected normal vectors) when the point cloud approximates a smooth manifold. A stability property has been proposed with respect to perturbations of point positions (Gaussian noise). We have also proposed a new set of estimators for computing differential properties on point clouds exploiting the full curvature tensor from the shape operator. We did not provide a convergence proof for these estimators but we show better experimental behaviors than the proven ones in quantitative studies, for comparable computational requirements.

Limitations. In order to estimate signed curvature information, our APSS approach requires oriented normal vectors. In most acquisition scenarios (e.g. Lidar, photogrammetry), normal vectors 
T. Lejemble \& D. Coeurjolly \& L. Barthe \& N. Mellado / Stable and efficient differential estimators on oriented point clouds

\begin{tabular}{llll}
\hline Name & Quantity & Ref. & Comment \\
\hline Distance to barycenter & Mean curvature & {$[$ PWY*07, Theorem 6] } & convergence proof \\
\hline Distance to PCA plane & Mean curvature & {$[$ DMSL11, Theorem 2] } & convergence proof \\
\hline Point Set Surfaces (PSS) & Curvature tensor & {$[$ ABCO*01] } & \\
Osculating Jets (OJets) & Curvature tensor & {$[$ PP05] } & convergence proof \\
Wavejets (WJets) & Curvature tensor & {$[\mathrm{BDC} 18]$} & \\
\hline Voronoi Covariance Measure (VCM) & Curvature tensor & {$[$ MOG10] } & convergence proof \\
\hline Algebraic Point Set Surfaces (APSS) & $\tilde{\mathbf{n}}($ Equation $(8)), \tilde{H}$ (Equation (7)) & {$[$ GG07] } & convergence proof (here) \\
Algebraic Shape Operator (ASO) & $\tilde{\mathbf{n}}_{w}, \tilde{\kappa}_{1}, \tilde{\kappa}_{2}, \tilde{H}_{w}, \tilde{K}_{w}($ Equations (31)-(33)) & here & \\
\hline
\end{tabular}

Table 1: Compared methods: list of estimators evaluated in our experiments (Sections 6.1 and 6.2).

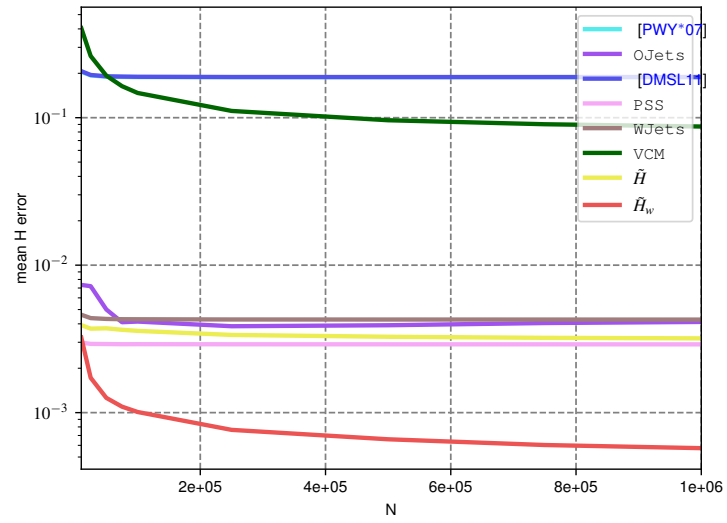

(a)

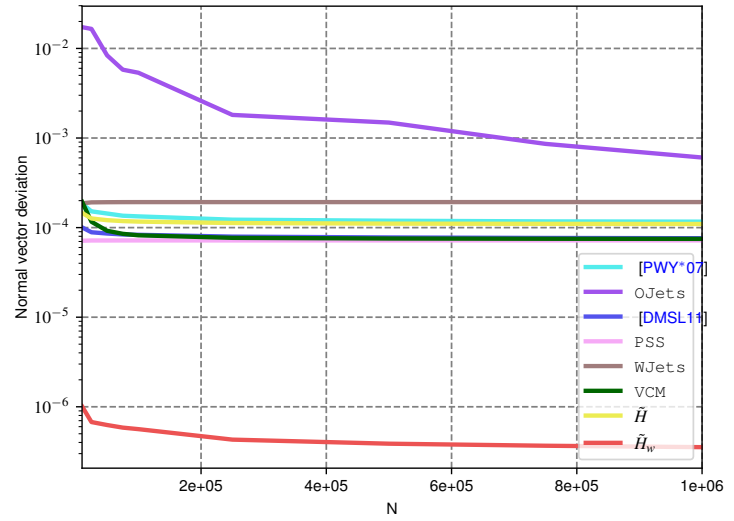

(b)

Figure 4: Asymptotic analysis: error plots in logscale of the absolute mean curvature $(a)$ and corrected normal vector errors $(b)$ as a function of the number of points $N$ (with fixed $r=0.01$ ).

are provided and oriented relatively to the acquisition device position. In our experimental evaluation, we demonstrate that perturbation in the normal vector orientation has limited impact on the estimated quantities to some extent.

Future work. A first challenging future work would be to extend the proposed framework to the non-oriented algebraic fitting procedure introduced by Chen et al. [CGBG13]. This would alleviate the normal vector orientation requirement when this information is not provided in the input point cloud. A more technical future work would be to extend the stability results by considering alternative noise models (e.g. Hausdorff perturbation, Lidar specific models, etc), or to study alternative reconstruction kernels [RJT*05, OGG09].

\section{References}

[AA03] AdAmson A., AleXa M.: Approximating and intersecting surfaces from points. In Proceedings of the 2003 Eurographics/ACM SIGGRAPH Symposium on Geometry Processing (Goslar, DEU, 2003), SGP '03, Eurographics Association, p. 230-239. 2

[AA06] AdAmson A., AleXA M.: Anisotropic point set surfaces. In Proceedings of the 4th international conference on Computer graphics, virtual reality, visualisation and interaction in Africa (2006), pp. 7-13. 2
[ABCO*01] Alexa M., Behr J., Cohen-Or D., Fleishman S., LEVIN D., Silva C. T.: Point set surfaces. In Visualization Conference (2001), IEEE, pp. 21-29. 2, 3, 5, 8

[AGJ*21] Alliez P., Giraudot S., Jamin C., Lafarge F., Mérigot Q., Meyron J., Saboret L., Salman N., Wu S., YILDIRAN N. F.: Point set processing. In CGAL User and Reference Manual, 5.2.1 ed. CGAL Editorial Board, 2021 URL: https://doc.cgal.org/5.2.1/Manual/packages. html\#PkgPointSetProcessing 3.7

[AJB*07] Allaire S., Jace J.-J., Burdin V., Roux C., COUTure C.: Type-constrained robust fitting of quadrics with application to the $3 \mathrm{~d}$ morphological characterization of saddle-shaped articular surfaces. In IEEE 11th International Conference on Computer Vision (2007), pp. 18. doi:10.1109/ICCV.2007.4409163. 3

[AK04] Amenta N., KIL Y. J.: Defining point-set surfaces. In ACM SIGGRAPH 2004 Papers (New York, NY, USA, 2004), SIGGRAPH '04, Association for Computing Machinery, p. 264-270. doi:10.1145/ 1186562.1015713 .2

[BC94] Berkmann J., CAelli T.: Computation of surface geometry and segmentation using covariance techniques. IEEE Transactions on Pattern Analysis and Machine Intelligence 16, 11 (1994), 1114-1116. 3

[BDC18] BÉarzi Y., Digne J., Chaine R.: Wavejets: A local frequency framework for shape details amplification. Computer Graphics Forum 37, 2 (2018), 13-24. doi:10.1111/cgf.13338. 2, 8

[Ber48] BERTRAND J.: Démonstration d'un théorème de m. gauss. Journal de mathématiques pures et appliquées (1848), 80-82. 3 
T. Lejemble \& D. Coeurjolly \& L. Barthe \& N. Mellado / Stable and efficient differential estimators on oriented point clouds

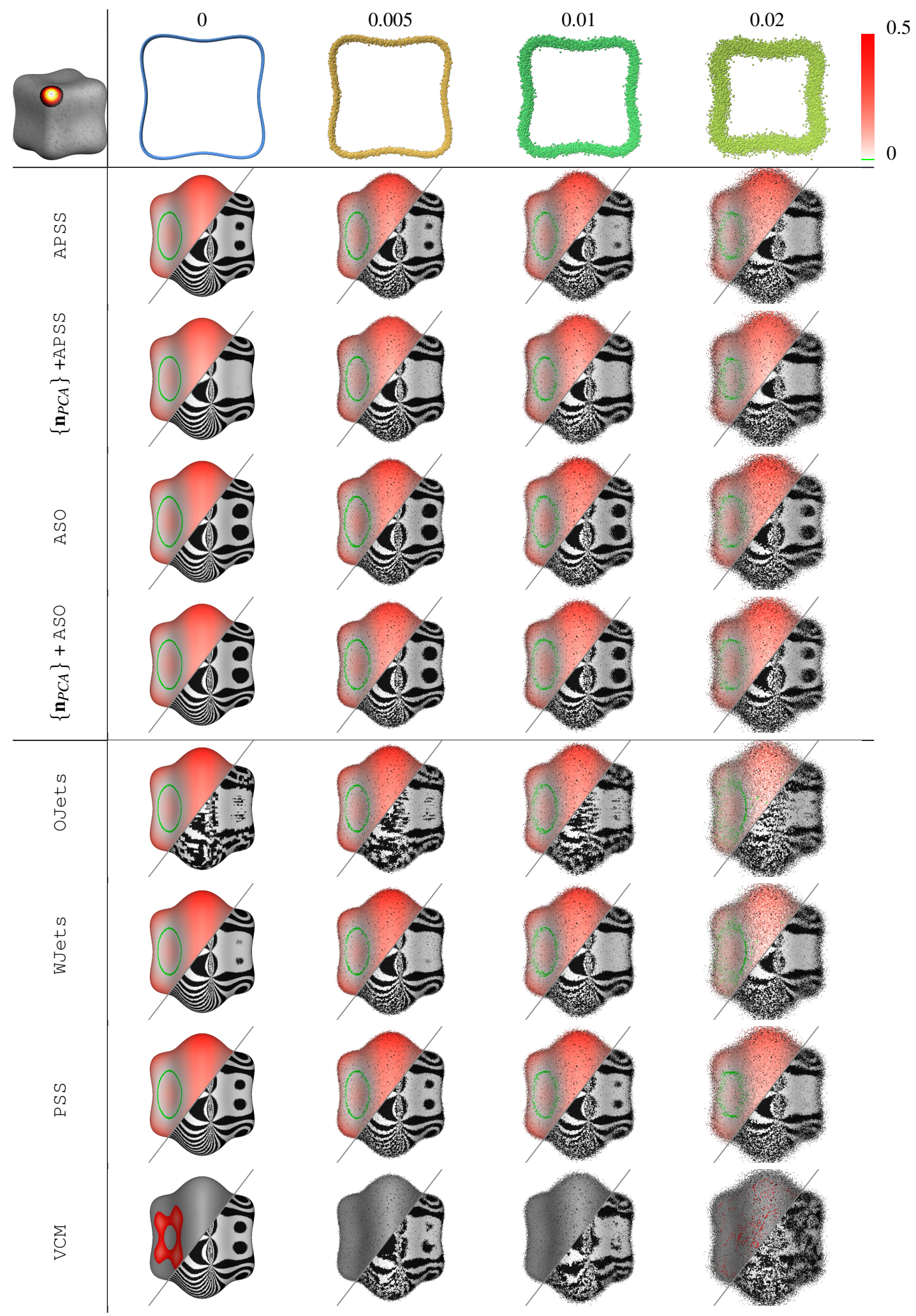

Figure 5: Stability with respect to noise on the positions: we evaluate the absolute value of the mean curvature and the corrected normal vectors for various noise level (Gaussian noise with standard deviations in $\{0,0.005,0.01,0.02\}$ ). Curvature measures are displayed using a white-red colormap with a green highlight for the zero-curvature values (grey values correspond to quantities outside the [0 : 0.5] range). To illustrate the smoothness of the corrected normal vectors, we consider a pure reflective material with a zebra like environment map to approximate isophotes.

(C) 2021 The Author(s)

Computer Graphics Forum $\odot 2021$ The Eurographics Association and John Wiley \& Sons Ltd. 
T. Lejemble \& D. Coeurjolly \& L. Barthe \& N. Mellado / Stable and efficient differential estimators on oriented point clouds

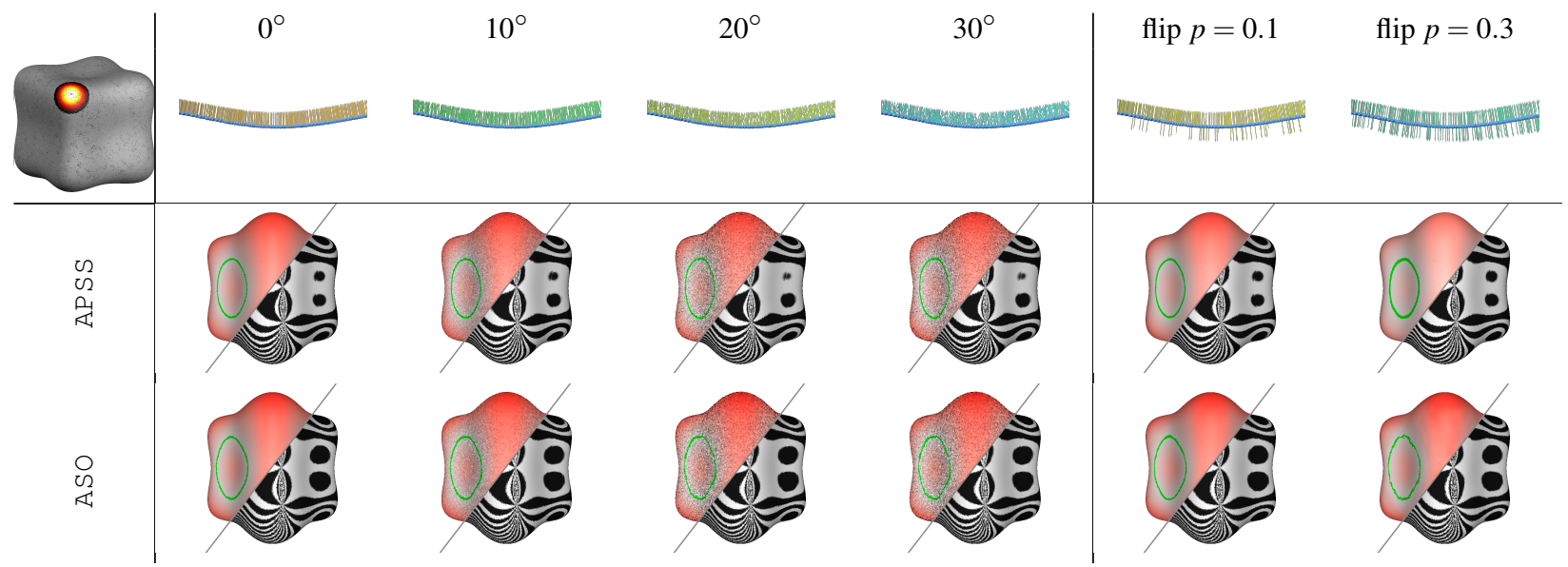

Figure 6: Stability of APSS and ASO on perturbed normal vector field input: using similar rendering as for Figure 5, we compared estimated quantities when normal vectors are perturbed using a random angular Gaussian noise of degrees in $\{0,10,20,30\}$ around the exact normal vectors, or when the normal vectors are flipped with probability 0.1 and 0.3 .

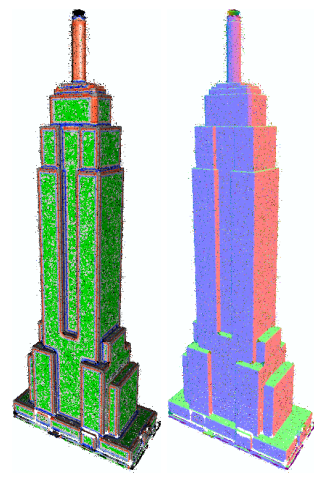

(a)

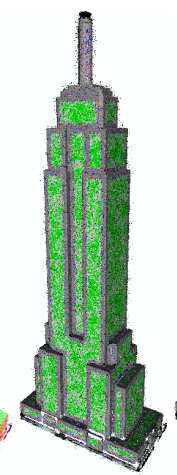

(c)

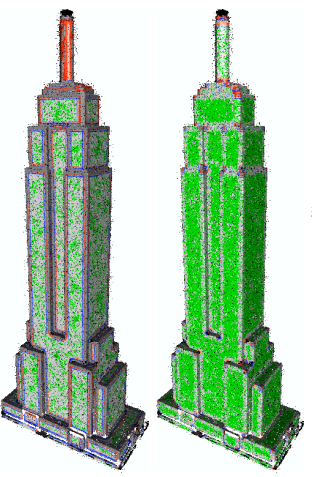

(d)

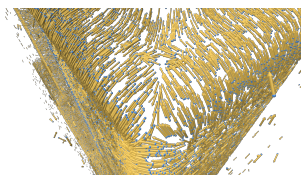

(f)

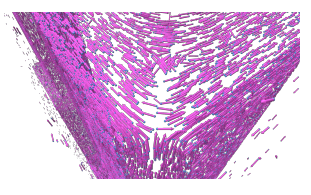

(g)

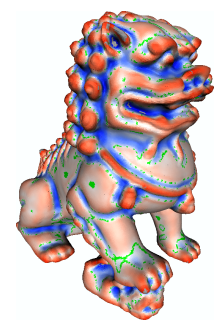

(h)

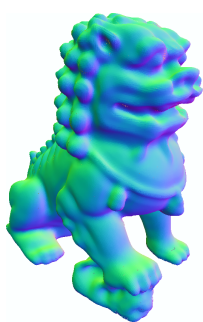

(i)

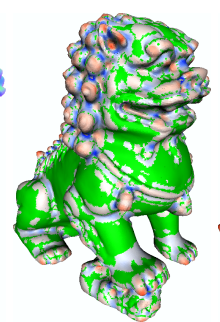

(j)

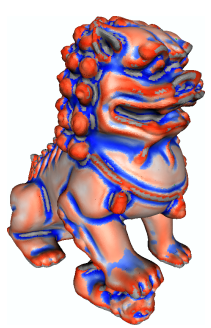

(k)

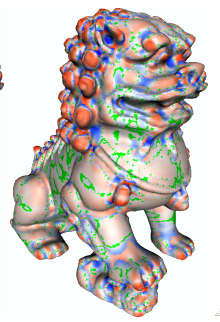

(1)

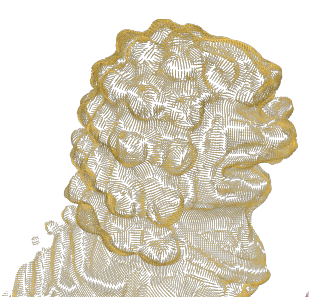

(m)

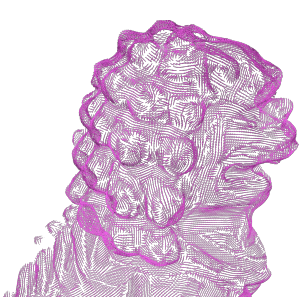

(n)

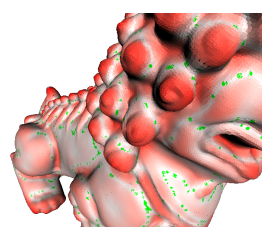

(o) APSS

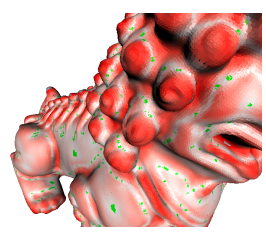

(p) $\mathrm{ASO}$

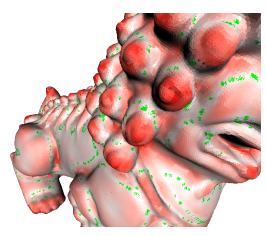

(q) OJets

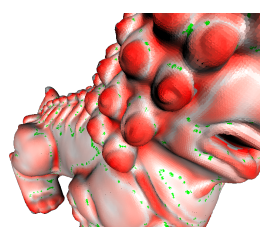

(r) P S S

Figure 7: Examples of differential quantities: on the Empire (951 600 points) and Dragon statue (127 572 points), from (a) to ( $g$ ) and (h) to $(n)$, the signed mean curvature, the corrected normal vectors, the first and second principal curvatures, the Gaussian curvature and the first and second curvature directions. On this smooth, noise-free shape, differences between APSS, ASO and OJets for instance are limited $((o-r))$. However, APSS and ASO are obtained about 10 times faster (seeTable 2). 
T. Lejemble \& D. Coeurjolly \& L. Barthe \& N. Mellado / Stable and efficient differential estimators on oriented point clouds

\begin{tabular}{lc|cc|cccccc}
\hline data & execution & {$\left[\right.$ PWY $\left.^{*} 07\right]$} & {$[$ DMSL11] } & APSS & ASO & OJet. & WJet S & PSS & VCM \\
\hline \multirow{2}{*}{ Dragon (Figure 7, 127K points) } & parallel & 0.07 & 0.08 & 0.08 & 0.24 & 0.98 & 1.21 & 0.55 & 14.31 \\
& sequential & 1.12 & 1.64 & 1.47 & 4.34 & 12.76 & 19.97 & 10.67 & 13.60 \\
\hline \multirow{2}{*}{ Pisa (Figure 1, 2.5M points) } & parallel & 1.64 & 2.37 & 2.31 & 7.82 & 22.75 & 39.41 & 20.21 & 307.94 \\
& sequential & 29.13 & 46.19 & 42.65 & 135.42 & 390.13 & 620.38 & 367.40 & 291.54 \\
\hline
\end{tabular}

Table 2: Timings: in seconds to compute the differential quantities with two execution approaches: parallel (OpenMP, 40 cores) and sequential.

[CGBG13] Chen J., Guennebaud G., Barla P., Granier X.: NonOriented MLS Gradient Fields. Computer Graphics Forum (2013). doi:10.1111/cgf.12164.8

[Cll13] Coeurjolly D., Lachaud J.-O., Levallois J.: Integral based curvature estimators in digital geometry. In Discrete Geometry for Computer Imagery (Berlin, Heidelberg, 2013), Gonzalez-Diaz R., Jimenez M.-J., Medrano B., (Eds.), Springer Berlin Heidelberg, pp. 215227. 3

[Con86] Connolly M. L.: Measurement of protein surface shape by solid angles. Journal of Molecular Graphics 4, 1 (1986), 3-6. doi: https://doi.org/10.1016/0263-7855 (86) 80086-8.2,3

[CP05] Cazals F., Pouget M.: Estimating differential quantities using polynomial fitting of osculating jets. Computer Aided Geometric Design 22, 2 (2005), 121 - 146. doi:https://doi.org/10.1016/j. cagd.2004.09.004.2,3,8

[CRT04] Clarenz U., RumpF M., Telea A.: Robust feature detection and local classification for surfaces based on moment analysis. IEEE Transactions on Visualization and Computer Graphics 10, 5 (2004), 516-524. doi:10.1109/TVCG.2004.34.3

[CSM03] COHEn-STEINER D., Morvan J.-M.: Restricted delaunay triangulations and normal cycle. In Proceedings of the Nineteenth Annual Symposium on Computational Geometry (New York, NY, USA, 2003), SCG '03, Association for Computing Machinery, p. 312-321. doi:10.1145/777792.777839. 3

[DC76] Do CARMO M. P.: Differential geometry of curves and surfaces. Prentice-Hall, 1976. 3

[DM14] Digne J., Morel J.-M.: Numerical analysis of differential operators on raw point clouds. Numerische Mathematik 127, 2 (2014), 255-289. 2, 3, 5

[DMSL11] Digne J., Morel J.-M., Souzani C.-M., Lartigue C.: Scale space meshing of raw data point sets. Computer Graphics Forum 30, 6 (2011), 1630-1642. doi:10.1111/j.1467-8659.2011. 01848 .x. $3,4,5,7,8,11$

[FCOAS03] Fleishman S., Cohen-Or D., Alexa M., Silva C. T.: Progressive point set surfaces. ACM Trans. Graph. 22, 4 (Oct. 2003), 997-1011. doi:10.1145/944020.944023.2

[GG07] Guennebaud G., Gross M.: Algebraic point set surfaces. ACM Trans. Graph. 26, 3 (July 2007), 23-es. doi:10.1145/ $1276377.1276406 .1,2,3,8$

[GGG08] Guennebaud G., Germann M., Gross M.: Dynamic sampling and rendering of algebraic point set surfaces. Computer Graphics Forum 27, 2 (2008), 653-662. doi:10.1111/j.1467-8659. 2008.01163 .х. 2,3

[GJ*10] Guennebaud G., JACOB B., ET AL.: Eigen v3. http://eigen.tuxfamily.org, 2010. 7

[GKOM18] Guerrero P., Kleiman Y., Ovsjanikov M., Mitra N. J.: Pcpnet learning local shape properties from raw point clouds. Computer Graphics Forum 37, 2 (2018), 75-85. do i : 10 .1111/cgf . 13343. 2,7

[GMgP05] Gelfand N., Mitra N. J., Guibas L. J., Pottmann H.: Robust global registration. In Proceedings of the Third Eurographics Symposium on Geometry Processing (Goslar, DEU, 2005), SGP '05, The Eurographics Association, p. 197-es. 2, 3
[HFG*06] Huang Q.-X., Flöry S., Gelfand N., Hofer M., PotTMAnN H.: Reassembling fractured objects by geometric matching. ACM Trans. Graph. 25, 3 (July 2006), 569-578. doi:10.1145/ $1141911.1141925 .2,3$

[HLP*20] Himeur C.-E., Lejemble T., Pellegrini T., Paulin M., Barthe L., Mellado N.: Pcednet: A neural network for fast and efficient edge detection in $3 \mathrm{~d}$ point clouds. arXiv preprint arXiv:2011.01630 (2020). 2, 3

[HT03] Hulin D., Troyanov M.: Mean curvature and asymptotic volume of small balls. The American Mathematical Monthly 110, 10 (2003), 947-950. 3

[KHS10] Kalogerakis E., Hertzmann A., Singh K.: Learning 3d mesh segmentation and labeling. ACM Trans. Graph. 29, 4 (July 2010). doi:10.1145/1778765.1778839.2

[KLM*13] Kim V. G., Li W., Mitra N. J., Chaudhuri S., DiVerdi S., FUnKhouser T.: Learning part-based templates from large collections of 3d shapes. ACM Trans. Graph. 32, 4 (July 2013). doi: $10.1145 / 2461912.2461933 .2$

[KSNS07] Kalogerakis E., Simari P., Nowrouzezahrai D., SINGH K.: Robust statistical estimation of curvature on discretized surfaces. In Proceedings of the Fifth Eurographics Symposium on Geometry Processing (Goslar, DEU, 2007), SGP '07, The Eurographics Association, p. 13-22. 2

[Lev98] LEVIN D.: The approximation power of moving least-squares. Mathematics of computation 67, 224 (1998), 1517-1531. 2

[Lev04] Levin D.: Mesh-independent surface interpolation. In Geometric modeling for scientific visualization. Springer, 2004, pp. 37-49. 2

[Lev15] LEVy B.: Geogram, 2015. 6

[LMBM20] Lejemble T., Mura C., Barthe L., Mellado N.: Persistence analysis of multi-scale planar structure graph in point clouds Computer Graphics Forum 39, 2 (2020), 35-50. doi : https : / / doi. org/10.1111/cgf.13910.3

[LT90] Liang P., TOdhunter J. S.: Representation and recognition of surface shapes in range images: A differential geometry approach. Comput. Vision Graph. Image Process. 52, 1 (Aug. 1990), 78-109. do i : $10.1016 / 0734-189 \times(90) 90124-E .3$

[LZH*07] LaI Y.-K., ZHOU Q.-Y., HU S.-M., WALlner J., PotTmAnN H.: Robust feature classification and editing. IEEE Transactions on Visualization and Computer Graphics 13, 1 (2007), 34-45. doi:10.1109/TVCG.2007.19.3

[MDS15] Mellado N., Dellepiane M., Scopigno R.: Relative scale estimation and $3 \mathrm{~d}$ registration of multi-modal geometry using growing least squares. IEEE Transactions on Visualization and Computer Graphics 22, 9 (2015), 2160-2173. 2, 3

[MGB*12] Mellado N., Guennebaud G., Barla P., Reuter P., SCHLICK C.: Growing least squares for the analysis of manifolds in scale-space. Computer Graphics Forum 31, 5 (2012), 1691-1701. doi : $10.1111 / j .1467-8659.2012 .03174$.x. 2, 3, 4, 5

[MHYS04] Manay S., Hong B.-W., Yezzi A. J., Soatto S.: Integral invariant signatures. In European Conference on Computer Vision (ECCV) (2004), Springer, pp. 87-99. 2, 3

[MLGB20] Mellado N., Lejemble T., Guennebaud G., Barla 
P.: Ponca: a point cloud analysis library, 2020. URL: https:// github.com/poncateam/ponca/. 7

[MOG10] Mérigot Q., OvsJanikov M., Guibas L. J.: Voronoibased curvature and feature estimation from point clouds. IEEE Transactions on Visualization and Computer Graphics 17, 6 (2010), 743-756. 2,8

[MrB*] Mourglia C., Roussellet V., Barthe L., Mellado N., Mathias P., VANDERHAEghe D., ET AL.: Radium engine. URL: https://github.com/STORM-IRIT/Radium-Engine/. 7

[NGM14] Nader G., Guennebaud G., Mellado N.: Adaptive multi-scale analysis for point-based surface editing. Computer Graphics Forum 33, 7 (2014), 171-179. doi:10.1111/cgf.12485. 3

[OGG09] ÖzTiReli A. C., Guennebaud G., Gross M.: Feature preserving point set surfaces based on non-linear kernel regression. Computer Graphics Forum 28, 2 (2009), 493-501. doi : https://doi. org/10.1111/j.1467-8659.2009.01388.x. 8

[PC21] Pouget M., CaZals F.: Estimation of local differential properties of point-sampled surfaces. In CGAL User and Reference Manual, 5.2.1 ed. CGAL Editorial Board, 2021. URL: https: // doc.cgal. org/5.2.1/Manual/packages.html\#PkgJetFitting3. 6,

[PGK02] Pauly M., Gross M., Kobbelt L. P.: Efficient simplification of point-sampled surfaces. In IEEE Visualization, 2002. VIS 2002. (2002), IEEE, pp. 163-170. 2, 3

[PKG03] PAuly M., Keiser R., Gross M.: Multi-scale feature extraction on point-sampled surfaces. Computer Graphics Forum 22, 3 (2003), 281-289. doi:10.1111/1467-8659.00675.2,3

[Pra87a] PRATT V.: Direct least-squares fitting of algebraic surfaces. SIGGRAPH Comput. Graph. 21, 4 (Aug. 1987), 145-152. doi: 10 . $1145 / 37402.37420 .2$

[Pra87b] PRATT V.: Direct least-squares fitting of algebraic surfaces. SIGGRAPH Comput. Graph. 21, 4 (Aug. 1987), 145-152. doi:10 . $1145 / 37402.37420 .3$

[PWhY09] Pottmann H., Wallner J., Huang Q.-X., Yang Y.L.: Integral invariants for robust geometry processing. Computer Aided Geometric Design 26, 1 (2009), 37 - 60. doi: https://doi .org/ $10.1016 / j$. cagd.2008.01.002.2,3

[PWY*07] Pottmann H., Wallner J., YANG Y.-L., LAI Y.-K., Hu S.-M.: Principal curvatures from the integral invariant viewpoint. Computer Aided Geometric Design 24, 8 (2007), 428 - 442. Discrete Differential Geometry. doi:https://doi.org/10.1016/j.cagd. $2007.07 .004 .3,7,8,11$

[QSMG17] Qi C. R., Su H., Mo K., Guibas L. J.: Pointnet: Deep learning on point sets for $3 \mathrm{~d}$ classification and segmentation. In Proceedings of the IEEE conference on computer vision and pattern recognition (2017), pp. 652-660. 2

[RGRG15] Ridel B., Guennebaud G., Reuter P., Granier X.: Parabolic-cylindrical moving least squares surfaces. Computers \& Graphics 51 (2015), 60 - 66. International Conference Shape Modeling International. doi:https://doi.org/10.1016/j.cag. 2015.05 .006 .2

[RJT*05] Reuter P., Joyot P., TRunZler J., Boubekeur T., SCHLICK C.: Surface reconstruction with enriched reproducing kernel particle approximation. In Proceedings Eurographics/IEEE VGTC Symposium Point-Based Graphics, 2005. (2005), pp. 79-87. doi: $10.1109 /$ PBG.2005.194068.8

[TAM*19] Tachella J., Altmann Y., Mellado N., Mccarthy A., Tobin R., Stuart Buller G., Tourneret J.-Y., MclaughLIN S.: Real-time 3D reconstruction from single-photon lidar data using plug-and-play point cloud denoisers. Nature Communications 10 (2019), 4984. doi:10.1038/s41467-019-12943-7. 2

[TGDM18] Thomas H., Goulette F., Deschaud J.-E., MarCOTEGUI B.: Semantic classification of $3 \mathrm{~d}$ point clouds with multiscale spherical neighborhoods. In 2018 International Conference on $3 D$ Vision (3DV) (2018), IEEE, pp. 390-398. 2
[YLHP06] Yang Y.-L., LaI Y.-K., Hu S.-M., PotTManN H.: Robust principal curvatures on multiple scales. In Proceedings of the Fourth Eurographics Symposium on Geometry Processing (Goslar, DEU, 2006), SGP '06, The Eurographics Association, p. 223-226. 3

[YQ07] YANG P., QIAN X.: Direct computing of surface curvatures for point-set surfaces. SPBG 7 (2007), 29-36. 2 